\title{
New elastomeric silicone based networks applicable as electroactive systems
}

Bejenariu, Anca Gabriela; Boll, Mads; Lotz, Mikkel Rønne; Vraa, Christoffer; Skov, Anne Ladegaard

Published in:

Proceedings of SPIE - The International Society for Optical Engineering

Link to article, DOI:

$10.1117 / 12.876983$

Publication date:

2011

Document Version

Publisher's PDF, also known as Version of record

Link back to DTU Orbit

Citation (APA):

Bejenariu, A. G., Boll, M., Lotz, M. R., Vraa, C., \& Skov, A. L. (2011). New elastomeric silicone based networks applicable as electroactive systems. Proceedings of SPIE - The International Society for Optical Engineering, 7976(76762V). https://doi.org/10.1117/12.876983

\section{General rights}

Copyright and moral rights for the publications made accessible in the public portal are retained by the authors and/or other copyright owners and it is a condition of accessing publications that users recognise and abide by the legal requirements associated with these rights.

- Users may download and print one copy of any publication from the public portal for the purpose of private study or research.

- You may not further distribute the material or use it for any profit-making activity or commercial gain

- You may freely distribute the URL identifying the publication in the public portal 


\title{
New elastomeric silicone based networks applicable as electroactive systems
}

\author{
Anca G. Bejenariu, Mads Boll, Mikkel R. Lotz, Christoffer Vraa, and Anne L. Skov* \\ Department of Chemical and Biochemical Engineering, DTU
}

\begin{abstract}
Commercial elastomer materials are available for dielectric electroactive polymer (DEAP) purposes but the applied commercial elastomers have not been developed with the specific application in mind. It is therefore obvious that optimization of the elastomer material should be possible. In this study we focus on optimization of the mechanical properties of the elastomer and show that it is possible to lower the elastic modulus and still not compromise the other required mechanical properties such as fast response, stability, low degree of viscous dissipation and high extensibility.

The elastomers are prepared from a vinyl-terminated polydimethyl siloxane (PDMS) and a 4-functional crosslinker by a platinum-catalyzed hydrosilylation reaction between the two reactants. Traditionally, elastomers based on hydrosilylation are prepared via a 'one-step two-pot' procedure (with a mix A and a mix B mixed in a given ratio). An alternative network formulation method is adopted in this study in order to obtain an elastomeric system with controlled topology - a so-called bimodal network. Bimodal networks are synthesized using a 'two-step four-pot' mixing procedure which results in a nonhomogeneous network structure which is shown to lead to novel mechanical properties due to the low extensibility of the short chains and the high extensibility of the long chains. The first ensures stability and the last retards the rupture process thereby combining two desired properties for DEAP purposes without necessarily compromising the viscous dissipation.
\end{abstract}

Several elastomers are prepared and tested for the linear viscoelastic behaviour, i.e. behaviour in the small-strain limit (up to approximately $10 \%$ strain). The bimodal networks are, however, capable of extensions up to several times their initial length but the focus here is the small-strain limit.

Keywords: silicone, bimodal networks, mechanical properties, mechanical stability, rheology, elastic modulus

\section{INTRODUCTION}

Electroactive polymers (EAPs) have attracted increasing attention as potential candidates for artificial muscles and are soft, flexible materials characterized by lightweight, flexibility, toughness and shape-processability, with responsive and tuneable properties ${ }^{1-4}$. Amongst electronic EAPs, the dielectric elastomers (DE) have a good combination of electromechanical properties such as high achievable strains and stresses, fast response speeds, long lifetime, high reliability and high efficiency ${ }^{5}$. DEs are attractive polymers due to the relative ease of determining a mechanical response upon low electrical stimulation (under several volts). The critical components for practical dielectric elastomer actuator design are the dielectric materials, the formulation and the processing ${ }^{6}$ (single or folded sheet, multilayer, etc. ${ }^{7}$ ).

While several types of EAPs have been investigated ${ }^{3,8-10}$ the electroelastomers, also known as dielectric elastomers, have been particularly attractive for large strain and high-power applications ${ }^{5,11,12}$. Attention has been paid lately to the silicone and acrylic based soft elastomers in the field of new actuator technology. $\mathrm{VHB}^{\mathrm{TM}}$ acrylic elastomers from $3 \mathrm{M}$ have been widely used in actuators and have shown good performance by producing large strains. Silicone rubber films based on a poly(dimethylsiloxane) (PDMS) backbone have been also used with good results as electrically responsive materials ${ }^{13}$. However, the commercial availability of silicones to DE is limited to a very small number of materials (e.g. CF19-2186 from Nusil and KE441 from ShinEtsu) with few possibilities for optimization to specific uses since the only variable is the ratio of mixing the parts in two-part systems.

Electroactive Polymer Actuators and Devices (EAPAD) 2011, edited by Yoseph Bar-Cohen, Federico Carpi, Proc. of SPIE Vol. 7976, 79762V · (c) 2011 SPIE · CCC code: 0277-786X/11/\$18 · doi: 10.1117/12.876983 
Viscoelasticity is a mechanism that plays a significant role in the performance and reliability of the DEAPs ${ }^{14}$. For reliability of the DEAP a displacement attenuation can not be accepted. Several failure models of the DEAP also depend on the viscoelastic behaviour of the elastomer ${ }^{15}$. Elastomers are usually characterized mechanically in terms of the nominal stress $\mathrm{f}^{*}=\mathrm{f} / \mathrm{A}$, where $\mathrm{A}$ is the cross-sectional area ${ }^{16}$, but also commonly in terms of the reduced stress defined as $\left[f^{*}\right]=f^{*} /\left(\alpha-\alpha^{-2}\right)$ (Figure 2$)$, where $\alpha$ represents the elongation or relative length of the sample. The latter expression allows for a direct comparison to e.g. the Mooney Rivlin model ${ }^{17}$ whereas the first representation gives a clearer picture of the different regimes involved due to the different slopes. However, exact and reproducible elongational measurements are difficult and time-consuming for soft networks ${ }^{18,19}$ whereas a linear visco-elastic (LVE) diagram can be obtained easily from commercial rheometers. The LVE diagram provides direct information on important properties, namely the elastic modulus and the degree of viscous dissipation within the system, which will cause significant displacement attenuation and unfavourable heating of the material.

A polymer network is a three-dimensional entity formed by the interconnection of polymer chains and is sometimes referred to as an elastomer in the case of highly elastic material. The connectivity of the macromolecular chains might be more or less complete, depending on the used stoichiometry ratio between the reactive groups. Polymer networks with incomplete structure contain several structures, namely 1) the elastically active network chains, 2) dangling chains (and to a smaller extent dangling substructures) and 3) a so-called sol fraction which is not attached chemically to the network $^{20}$. Most soft elastomers are prepared by applying a random crosslinking process of an endlinked functional polymer with a crosslinker of a given functionality $f$, and it is referred to as unimodal network. The elastic modulus depends on the mass ratio between the aforementioned network fractions (which are governed by the stoichiometry) but also on the length of the polymer and to a lesser extent on the functionality of the crosslinker ${ }^{17}$. The dangling chains and the sol fraction are contributing to a lowering of the elastic modulus but also give rise to unwanted relaxation mechanisms causing undesired viscous dissipation. This can be observed from an LVE-diagram as an increase in the storage modulus $\left(G^{\prime}\right)$ with increasing frequency. Furthermore the materials tend to become sticky and difficult to handle. Therefore a decrease of the elasticity caused by a stoichiometric imbalance far from 1 is not a viable path for DEAP applications.

Alternatively the modulus can be lowered by increasing the molecular weight of the polymer and hence increase the distance between crosslinkers. The lowering effect is, however, limited by the molecular entanglement weight of the polymer, so the effect is quite limited for soft elastomers where the molecular weight already is approaching the molecular entanglement weight. The limiting effect of the entanglements can be partly excluded by an altered preparation procedure but the resulting materials become very fragile and with slow response time ${ }^{21}$.

Another approach is to play with heterogeneity effects arising from controlled reactions of the elastomers. An example is the so-called bimodal networks which are based on the same constituents as traditional networks and -generally- in the same amounts but where several steps of mixing allow for formation of clusters and therefore the heterogeneity appears. The two types of elastomers are illustrated in Figures $1 \mathrm{a}$ and $1 \mathrm{~b}$.

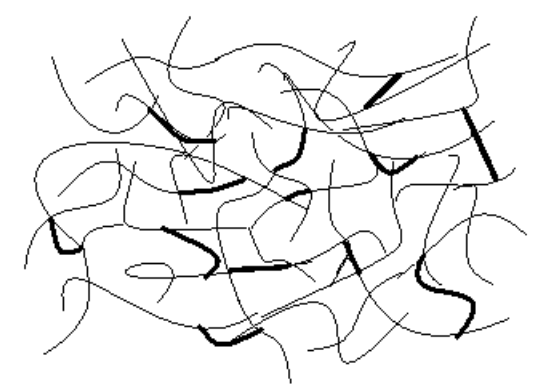

Figure 1a. Schematic illustration of a bimodal network with a non-controlled distribution of the short polymeric chains

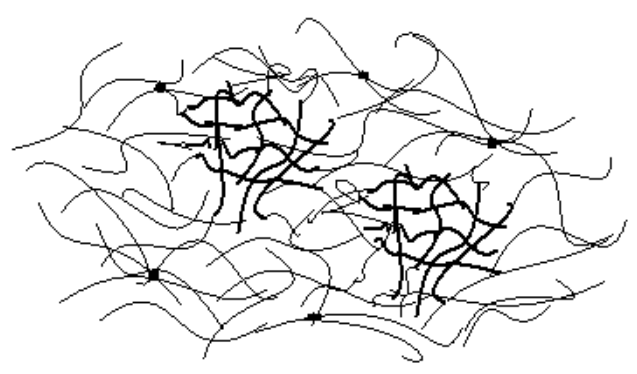

Figure 1b. Schematic illustration of a bimodal network with a controlled clustered distribution of the short polymeric chains

In Figure 2, typical variations of the nominal stress with elongation for unimodal and bimodal networks are shown ${ }^{16}$. The right ends of the curves illustrate when the materials rupture which is an important property in high-strain applications. 
The bimodal network should be imagined to consist of both the short-and the long chain polymers in Figure 2. If a simple two-pot mixing schema had been applied, it would have been expected that the network behaved as a weighted average of the two unimodal networks. It is, however, clear that at low strains the bimodal network resembles the shortchain system the most and at higher strains the behaviour resembles the long-chain system the most. Furthermore it is also observed that the bimodal network can be strained further than even the long-chain system before rupture which is counter-intuitive but nevertheless a proven property ${ }^{16}$.

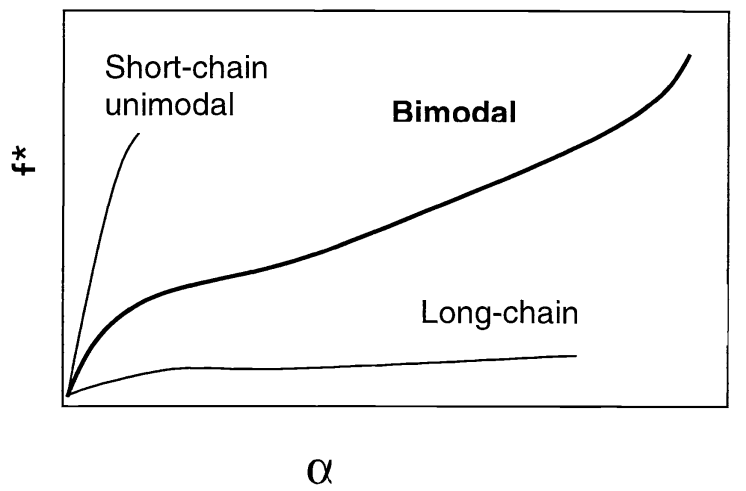

Figure 2. Typical dependence of nominal stress $\left(f^{*}\right)$ on elongation $(\alpha)$ for unimodal and bimodal networks at a given strain rate ${ }^{16}$

In this study different uni- and bimodal networks are prepared and the rheological properties are investigated as function of the stoichiometric imbalance and the relative weight fractions of short and long chains. Furthermore two types of long chains have been applied to investigate the effect on the elastic modulus and the viscous dissipation.

\section{MATERIALS AND METHODS}

Both unimodal and bimodal networks are prepared using polydimethyl siloxane (PDMS) chains crosslinked with a 4functional hydride crosslinker. The crosslinking reaction taking place is presented in Schema 1.

The network samples are synthesized using different molecular weight polydimethyl siloxanes (PDMS): DMS-V05 with $\mathrm{M}_{\mathrm{n}}=800 \mathrm{~g} / \mathrm{mol}$, DMS-V35 with $\mathrm{M}_{\mathrm{n}}=49500 \mathrm{~g} / \mathrm{mol}$ and DMS-V41 with $\mathrm{M}_{\mathrm{n}}=62400 \mathrm{~g} / \mathrm{mol}$ and a low molecular weight crosslinker: tetrakis(dimethyl siloxy)-silane with $\mathrm{M}_{\mathrm{w}}=328 \mathrm{~g} / \mathrm{mol}$. All the polymers are purchased from Gelest Inc. and the applied molecular weights are supplied by the company. The catalyst platinum, cyclovinylmethyl-siloxane complex (511) is provided by Hanse Chemie.

The final networks prepared as thin films are stored between $50 \mu \mathrm{m}$ thickness ethylen-tetrafluorethylen (ETFE) foils and kept in a dry place until use.

In general the unimodal and the bimodal networks are prepared by using 2 premixes or 4 pre-premixes, respectively. The purpose of the premix or the pre-premix preparation is to ensure sufficient mixing of catalyst and polymer, and crosslinker and polymer, respectively and also to control the final reaction ${ }^{17}$ Finally, the premixes or pre-premixes are mixed in different mass ratios corresponding to different stoichiometric values, $r$. The stoichiometry imbalance, $r$, is defined as the relationship between the reactive groups of the PDMS and crosslinker, i.e. vinyl $\left(-\mathrm{CH}=\mathrm{CH}_{2}\right)$ and hydride $(-\mathrm{Si}-\mathrm{H})$ respectively ${ }^{19}$.

The two different unimodal networks samples are synthesized PDMS-V35 and PDMS-V41 and the 4-functional crosslinker, using the 'one-step two-pot' procedure. The premix A contains PDMS and catalyst, while the premix B contains PDMS and crosslinker. The unimodal networks are prepared by mixing the premixes A and B in different mass ratios. The reaction takes place at ambient conditions for at least 5 hours. 


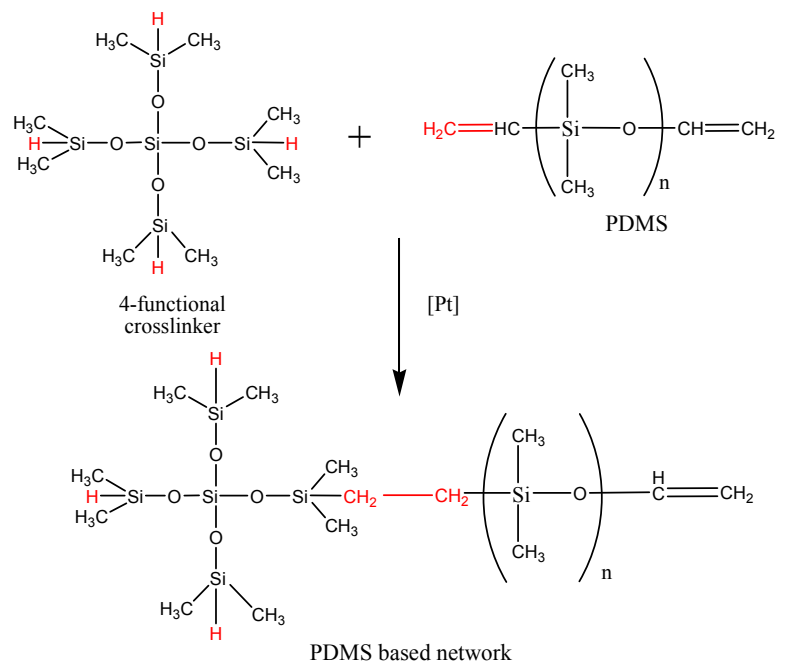

Schema 1. The crosslinking reaction between PDMS and the 4-functional crosslinker

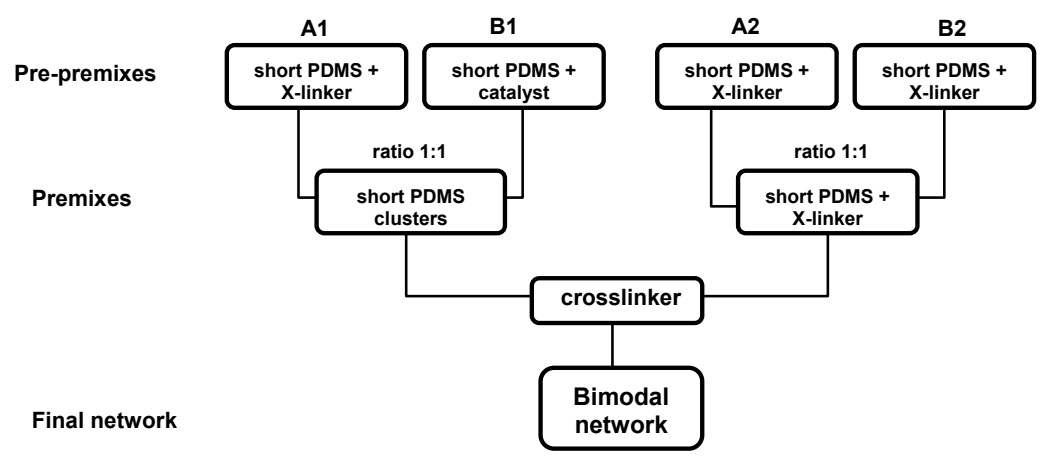

Figure 3. Conceptualization of the 'two-step four-pot' synthesis procedure of the bimodal networks

The bimodal networks are prepared using a 'two-step four-pot' mixing procedure. The schematic conceptualization of the procedure is presented in Figure 3. Four pre-premixes are prepared: 2 pre-premixes with short PDMS chains (PDMSV05) (A1 and B1) and 2 pre-premixes with long PDMS chains (PDMS-V35 or PDMS-V41) (A2 and B2). In the first step the pre-premixes are mixed two by two in ratios 1:1 which is corresponding to $r$ values of 0.30 . This $r$ value is chosen below the gel point of the system $\left(r_{c}=0.33\right)$ to avoid the formation of a network before the following step, but close enough to the $r_{c}$ so the short PDMS based clusters can form. In the second step the two premixes are mixed in certain amounts according to the final mass ratio between the short and the long chains (short:long = 97:3, 90:10, 80:20 or 3:97) and a calculated quantity of crosslinker is added in order to have a final bimodal network with a certain overall $\mathrm{r}$ value.

Films with a thickness of $100 \mu \mathrm{m}$ are prepared using an in-house constructed coating device (Figure 4 left). The coating device consists of a stainless steel plate and a knife with 2 predefined coating heights $(200$ and $100 \mu \mathrm{m})$; the error of the device is $\pm 0.1 \mu \mathrm{m}$ (Figure 4 right). The final mix of the reactants is poured on the ETFE foil and the knife is moved to form the film. After the network is cured over night, a second ETFE foil is added on the thin film. The use of ETFE foil has the advantage of making the very thin films handable.

The thin films are characterized rheologically using a controlled stress rheometer (AR2000) from TA Instruments set to a controlled strain mode with $2 \%$ strain, which is ensured to be within the linear regime of the material based on an initial strain sweep test. The linear visco-elastic (LVE) data are measured with parallel-plate geometry of $25 \mathrm{~mm}$ in diameter at $25^{\circ} \mathrm{C}$. 

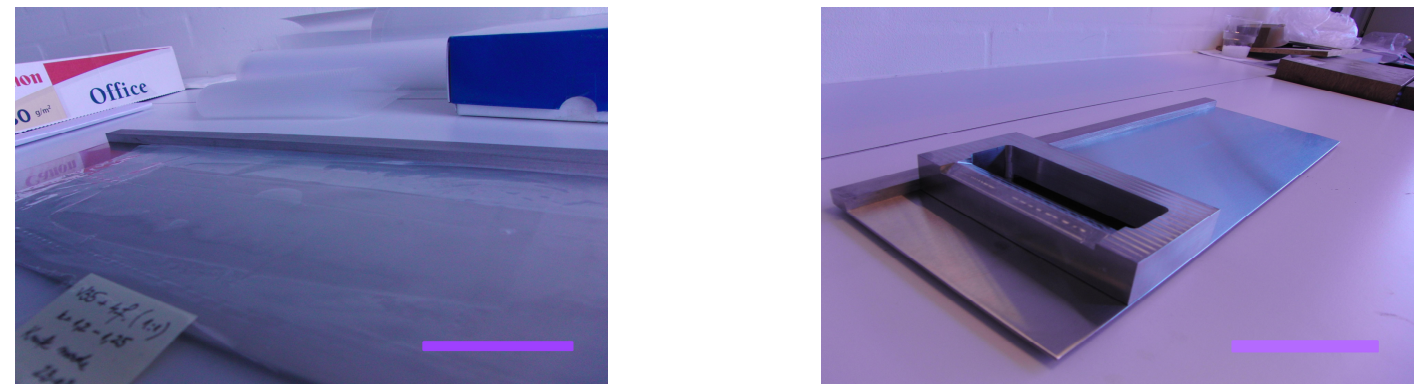

Figure 4. Photos of the $100 \mu \mathrm{m}$ thin film between the ETFE release liner (left) and the stainless steel knife (right)

Table 1. Summary of synthesized unimodal and bimodal networks. The first three columns refer to the weight percentages of the three different polymers. $r$ is the overall stoichiometric imbalance of the network, and $G_{o}$ is the storage modulus for the frequency approaching zero, which is related to the Young's modulus by $Y=3 \mathrm{G}_{0}$

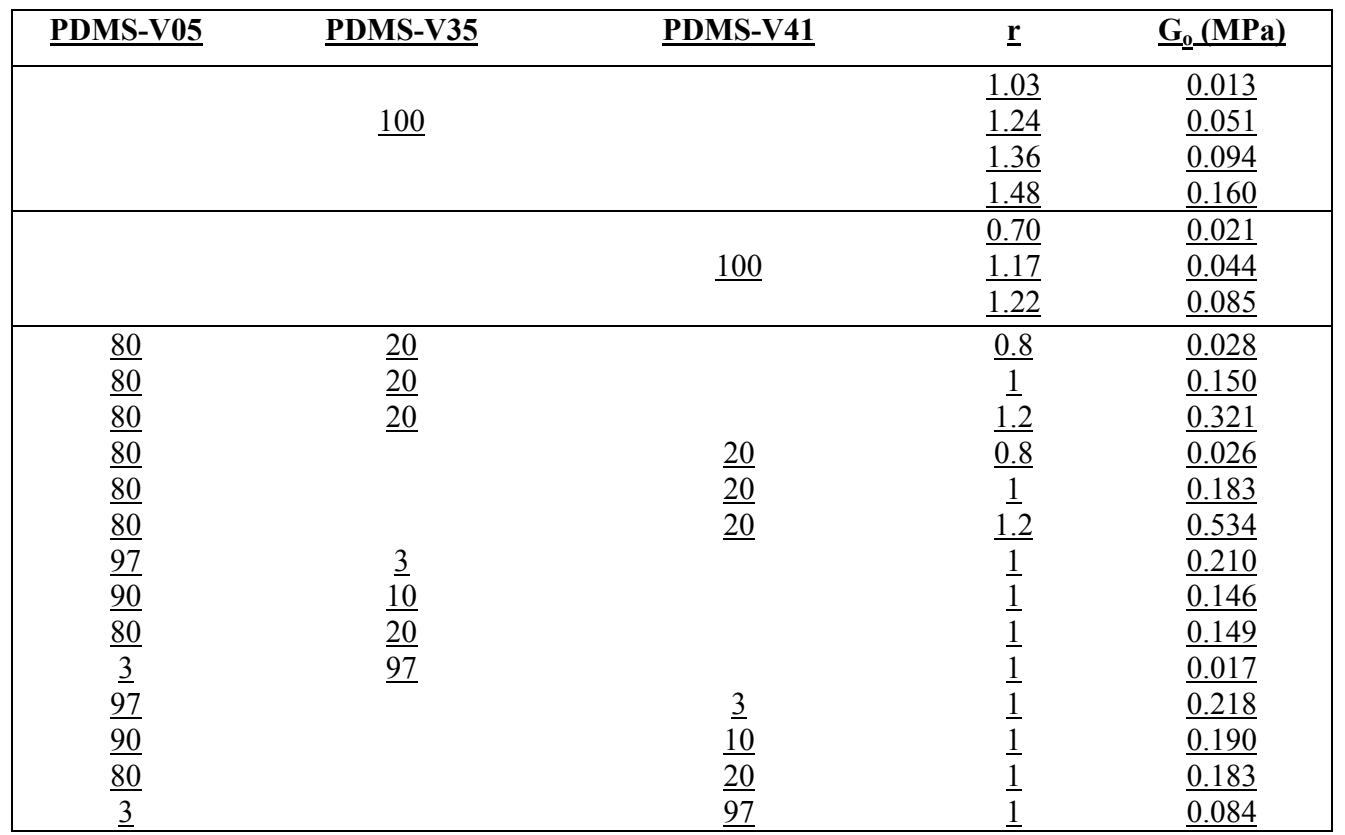

\section{RESULTS AND DISCUSSION}

In Figure 5 and Figure 6 the elastic behaviour as function of the applied frequency (LVE diagram) is shown for unimodal networks based on PDMS-V35 and PDMS-V41, respectively, with varying values of $r$. As the stoichiometric imbalance is increased, the elastic modulus is -as expected- increased as the fraction of elastically active chains is increased. It can furthermore be observed that with increasing $r$ the curves become more flat indicating a less pronounced effect of relaxation processes, which is also expected, as the sol and dangling chains fractions are both lowered with increasing $r$. There is only small deviation between the two different polymers. The PDMS-V41 networks (Figure 6) are characterized by slightly lower viscoelastic moduli values than the PDMS-V35 due to the higher molecular weight of the silicone (PDMS-V41) and thus to the increased distance between the crosslinking points in the network. The results of the elastic moduli are summarized in Table 1. 
Further, the bimodal networks are synthesized and characterized rheologically via the same frequency sweep procedure as the unimodal networks. The short PDMS chains used for the bimodal network formulation are the same in both systems (PDMS-V05), while the long PDMS chains are varied (V35 and V41, respectively).

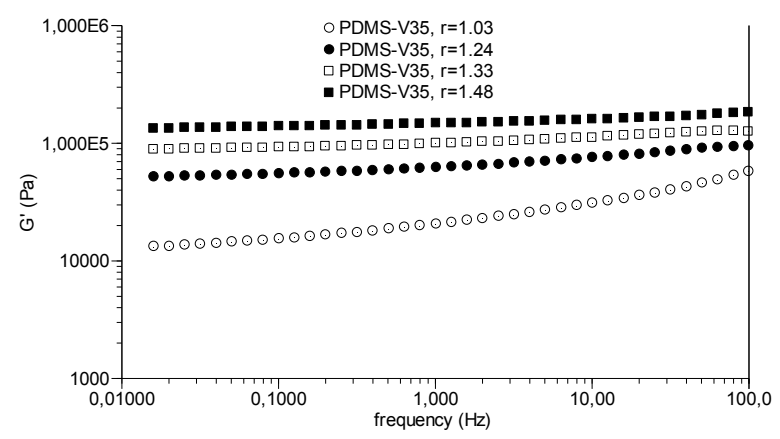

Figure 5. The viscoelastic behaviour of PDMS-V35 based unimodal network

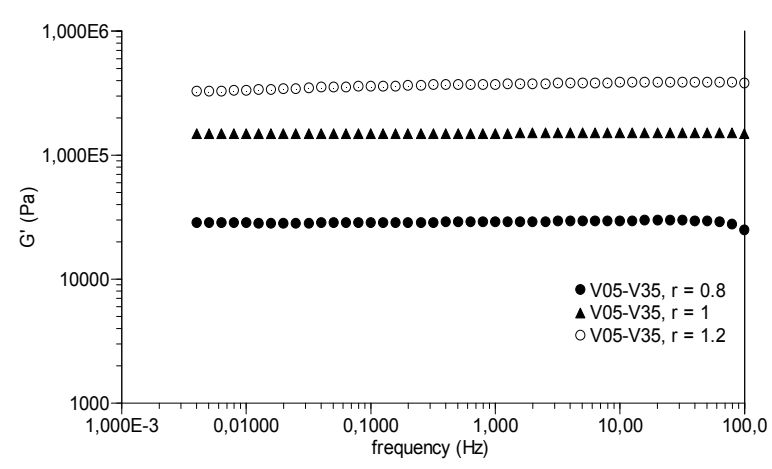

Figure 7. The viscoelastic behaviour of V05-V35 based network with a short:long mass ratio of 80:20 and different $r$ values

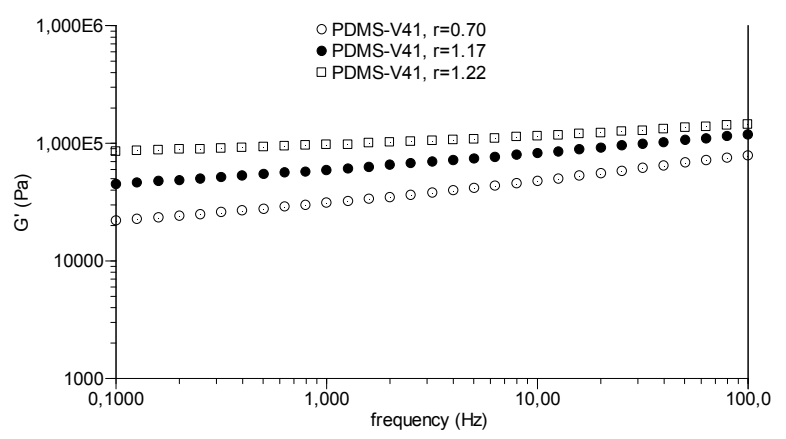

Figure 6. The viscoelastic behaviour of PDMS-V41 based unimodal network

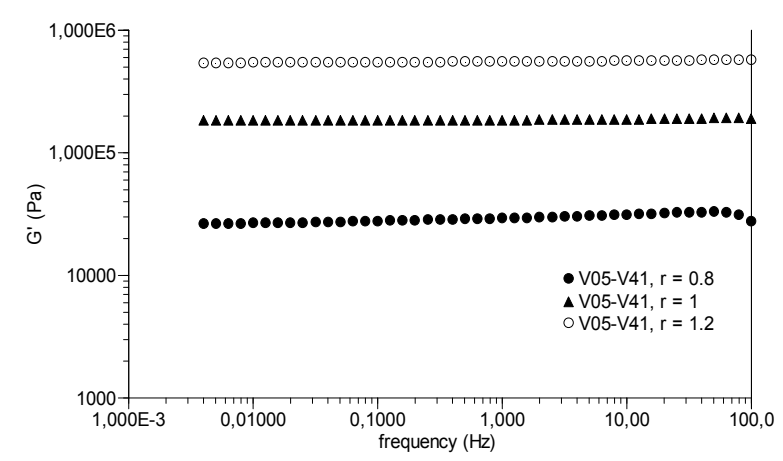

Figure 8. The viscoelastic behaviour of V05-V41 based network with a short:long mass ratio of 80:20 and different $r$ values

In Figure 7 and Figure 8 the frequency sweeps for PDMS-V35 and PDMS-V41 based bimodal networks, with mass ratios of 80:20 between the short and the long chains are shown for networks with varying stoichiometry. For both types of bimodal networks the values of the elastic moduli increase with increasing $r$ as expected. It is also evident that the tendency to viscous dissipation within the investigated frequency domain is limited even for networks with low elastic moduli. So, the introduction of the heterogeneity clearly leads to a non-contrary relation between low elastic modulus and little viscous dissipation. The low elastic moduli of the bimodal networks are very promising since as discussed in the introduction the bimodal networks will resemble the unimodal short-chain network the most in the low-strain limit (LVE limit) and hence it can be expected that the material will be even softer in the high-strain limit. This investigation will be performed in a future study. At the same time it can be noticed that there is not a significant difference between the two bimodal networks despite the molecular weight difference. 


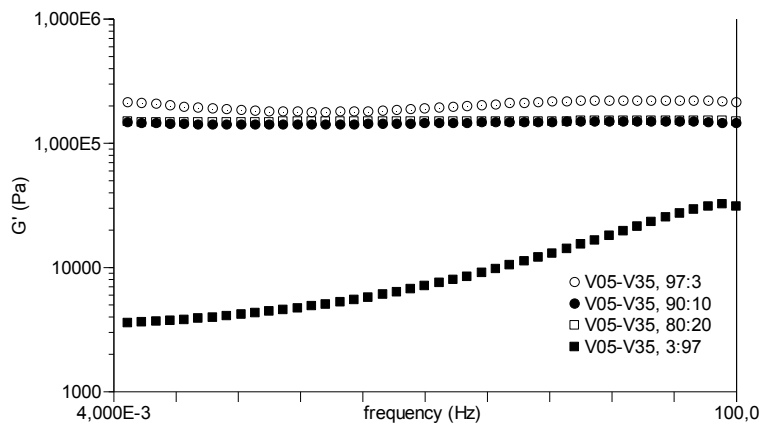

Figure 10. The viscoelastic behaviour of V05-V35 based network $(\mathrm{r}=1)$. The short:long chain ratio is varied

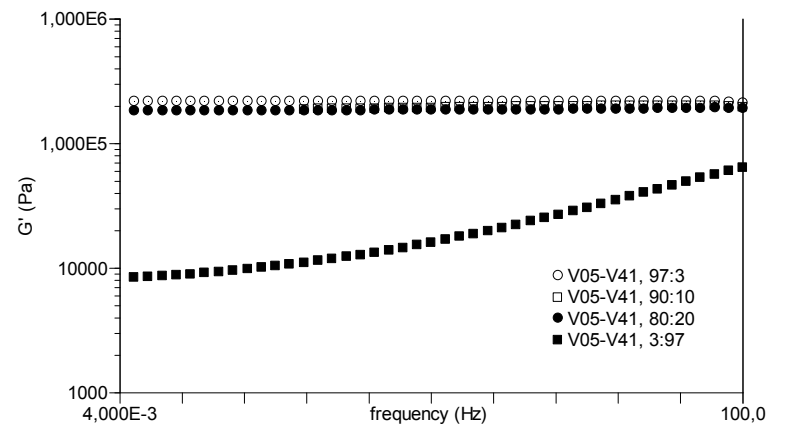

Figure 11. The viscoelastic behaviour of V05-V41 based network $(\mathrm{r}=1)$. The short:long chain ratio is varied

In figure 10 and Figure 11 the V05-V35 and V05-V41 based bimodal networks are compared with the mass ratio of short to long chains varying at stoichiometric conditions $(\mathrm{r}=1)$. This way the relative amounts of network fractions are identical for the systems but the crosslink density is varying. It is obvious that elastic modulus can be reduced by increasing the fraction of long polymer as the crosslink density will be decreased but the effect is very strong when the long polymer becomes the main reactant (by weight). The effect, however, seems rather insignificant at high mass ratios. The V05-V35 3:97 network (Figure 10) seems very promising since there is only little viscous dissipation and the elastic modulus is lowered by a factor of 10 compared to the unimodal network consisting of the long polymer without compromising significantly on the other mechanical properties.

\section{CONCLUSIONS}

Soft unimodal elastomers can be produced e.g. by increasing the molecular weight of the polymer or by a nonstoichiometric reaction but in both cases a significant viscous dissipation effect appears. The viscous dissipation effect can, however, to a large extent be limited if the elastomers are prepared by a 'two step four pot' procedure from two polymers, and at the same time a low elastic modulus can be obtained. The bimodal networks have promising properties for DEAP purposes as they are highly extendible, show little viscous dissipation, have a small elastic modulus, fast response, and have great stability.

The results in the article should be regarded as an initial study indicating several excellent properties of bimodal networks for DEAP applications. Further studies have to be performed in order to verify the applicability of the materials. Due to the complexity of the system there are many parameters to optimize, e.g. the stoichiometric imbalance in both prepremixes and in the final system, and the molecular weights of both polymers and the mixing scheme. 


\section{REFERENCES}

1. Baughman, R.H., Cui, C., Zakhidov, A.A., Iqbal, Z., Barisci, J.N., Spinks, G.M., Wallace, G.G., Mazzoldi, A., Rossi, D.D., Rinzler, A.G., Jaschinski, O., Roth, S. and Kertesz, M., "Carbon nanotube actuators ", Science 284, 1340-1344 (1999)

2. $\quad$ Pons, J.L., [Emerging Actuator Technologies: A Micromechatronic Approach], Chichester, (2005)

3. Bar-Cohen, Y., [Electroactive Polymer (EAP) Actuators as Artificial Muscles: Reality, Potential and Challenges], Bellingham, WA, (2004)

4. Madden, J.D., Vandesteeg, N.A., Anquetil, P.A., Madden, P.G.A., Takshi, A., Pytel, R.Z., Lafontaine, S.R., Wieringa, P.A. and Hunter, I.W., "Artificial muscle technology: Physical principles and naval prospects " IEEE J. Oceanic Eng. 29, 706-728 (2004)

5. Pelrine, R., Kornbluh, R., Pei, Q. and Joseph, J., "High-speed electrically actuated elastomers with strain greater than $100 \%$ " Science 287, 836-839 (2000)

6. Z Zhang, X., Lowe, C., Wissler, M., Jahne, B. and Kovacs, G., "Dielectric elastomers in actuator technology," Advanced engineering materials 7, (5), 361-367 (2005)

7. $\quad$ Sommer-Larsen, P. and Larsen, A.L., "Materials for dielectric elastomer actuators," Smart Structures and Materials 2004 Conference 5385, 68-77 (2004)

8. Pelrine, R., Eckerle, J. and Chiba, S., "Review of artificial muscle approaches," Third International Symposium on MicroMachine and Human Science, Nagoya, Japan, (1992)

9. Zhenyl, M., Scheinbeim, J.I., Lee, J.W. and Newman, B.A., "High-Field Electrostrictive Response Of Polymers," Journal of Polymer Science, Part B: Polymer Physics 32, 2721-2731 (1994)

10. Zhang, Q., Bharti, V. and Zhao, X., "Giant electrostriction and relaxor ferroelectric behavior in electronirradiated poly(vinylidene fluoride-trifluoroethylene) copolymer " Science 280, 2101-2104 (1998)

11. Pelrine, R., Kornbluh, R. and Kofod, G., Advanced Materials 12, 1223-1225 (2000)

12. Meijer, K., Rosenthal, M. and Full, R., "Muscle-like actuators? A comparison between three electroactive polymers. ," Smart Structures and Materials Conference, Newport Beach CA (2001)

13. Bar-Cohen, Y., Leary, S., Shahinpoor, M., Harrison, J. and Smith, J., "Electro-Active Polymer (EAP) actuators for planetary applications " Proc. SPIE 3669, 57-63 (1999)

14. Plante, J. and Dubowsky, S., "On the properties of dielectric elastomer actuators and their design implications," Smart Materials Structures 16, 227-236 (2007)

15. Plante, J. and Dubowsky, S., "Large-scale failure modes of dielectric elastomer actuators," International Journal of Solids and Structures 43, 7727-7751 (2006)

16. Mark, J.E., "Elastomers with multimodal distribution of network chain lengths," Macromolecular Symposia 191, 121-130 (2003)

17. Larsen, A., Sommer-Larsen, P. and Hassager, O., "Some experimental results for the end-linked PDMS network system," E-Polymers 0502-4 (2004)

18. Jensen, M.K., Rasmussen, H.K., Bach, A., Skov, A.L., Hassager, O. and Koldbech, H., "Planar Elongation of Soft Polymeric Networks," Rheologica Acta 49, 1-13 (2010)

19. Bejenariu, A., Rasmussen, H.K., Skov, A.L. and Hassager, O., "Large amplitude oscillatory extension of soft polymeric networks," Rheologica Acta 49, 807-814 (2010)

20. Larsen, A., Hansen, K., Sommer-Larsen, P., Hassager, O., Bach, A., Ndoni, S. and Jorgensen, M., "Elastic properties of nonstoichiometric reacted PDMS networks " Macromolecules 36, 10063-10070 (2003)

21. Larsen, A., Sommer-Larsen, P. and Hassager, O., "How to tune rubber elasticity," SPIE Proceedings 5385, 108$117(2004)$ 